\title{
MODIFICATION OF THE LEP ELECTROSTATIC SEPARATOR SYSTEMS FOR OPERATION WITH BUNCH TRAINS
}

\author{
B.Balhan, A.Burton, E.Carlier, J.-P.Deluen, J.Dieperink, N.Garrel, B.Goddard, R.Guinand, \\ W.Kalbreier, M.Laffin, M.Lamont, V.Mertens, J.Poole, H.Verhagen, CERN, CH-1211 Geneva
}

To meet the LEP2 luminosity requirements for W-pair production, it is planned to operate LEP with Bunch Trains from 1995 onwards. This new mode of operation entails significant modification both to the existing separator hardware and its control system. The changes have been implemented so as to provide maximum flexibility for the realisation of the Bunch Train scheme, and also make a return to operation with Pretzel separation possible during 1995. Two LEP Interaction Points (IP) were equipped with new separators in late 1994, enabling first tests with the collision of one train of four $\mathrm{e}^{+}$bunches with one train of four $\mathrm{e}^{-}$bunches. During the 1994/95 shutdown, four separators have been installed in the two remaining experimental IPs, and eight separators in the non-experimental IP have been displaced to new positions. Details are given of optics requirements for the separator installations, the polarity of the closed orbit separator bumps, system modifications, and performance considerations. Results are presented of investigations into the effects of separator polarity on High Voltage performance and on the commissioning of the new hardware and software systems during tests of the Bunch Train scheme in 1994.

\section{INTRODUCTION}

For Bunch Trains in LEP [1] the minimum inter-bunch spacing of $87 \lambda_{\mathrm{rf}}(74.2 \mathrm{~m}$ or $247.5 \mathrm{~ns})$ is imposed by the distance from the IP to the first electrostatic separator, with the maximum number of bunches in a train limited to four by the capabilities of the LEP experiments.

To operate LEP with Bunch Trains from 1995 onwards, additional separators have been installed around the even experimental IP, and changes to the separator installations have been made in the odd IPs. The controls software and hardware has been modified accordingly. The changes have been implemented so as to optimize flexibility for operation with Bunch Trains, streamline the controls of the various separator types, and to minimise the level of intervention should a return to Pretzel operation be necessary [2].

\section{OPTICS CONSIDERATIONS}

\section{A. Tests in 1994}

For the initial tests in 1994 [3] it was realised that the polarities of the bumps in IP 4 and 8 should be opposed, in order that the residual vertical dispersion be reduced to acceptable levels. With the convention that a positive $(+)$ bump is defined by an upwards deflection of $\mathrm{e}^{+}$in the first separator, the bump in IP4 was installed as positive, and that in IP8 negative. The tests were made with this configuration.

\section{B. Operation in 1995 and beyond}

With Bunch Train bumps in all four even experimental IP, numerical simulations show that two of the bumps should be positive and two negative. This combination minimizes both the vertical offsets and slope in the collision of the bunches of a given train, minimizes the residual vertical dispersion and maximizes the possible luminosity [4]. The bump polarities in the odd IPs must also alternate, with the constraints from the LEP beam dumping system fixing the bump in IP 5 to be positive. The variant shown in table 1 was eventually chosen.

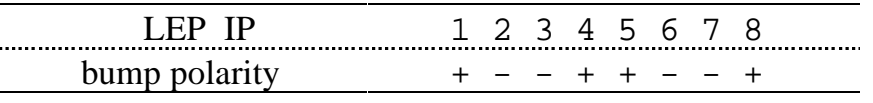

Table 1: Direction of separator Bunch Train bumps.

\section{SEPARATOR LAYOUTS}

The separation of a train of bunches requires that a sufficiently long orbit bump is created to separate the individual bunches at all parasitic encounters. In LEP, the minimum inter-bunch spacing of $74.2 \mathrm{~m}$ required the addition of two new separators per even IP, and the displacement of two existing separators per odd IP, to accommodate up to four bunches per train. The 1995 separator positions and $\mathrm{e}^{+} \mathrm{e}^{-}$ trajectories are illustrated for an experimental (even) IP and an odd IP in figures 1 and 2, respectively.

In the even IP, the newly constructed vertical separators (ZL) were placed near quadrupoles QS7 to provide the initial deflection for the separation bump. These separators are powered by the high voltage (HV) generators of the Pretzel ZX separators, with one electrode at $\mathrm{HV}$ and one at ground. In the odd IP, the outer separators have been moved further from the IP, to positions near quadrupoles QL8.

The existing horizontal Pretzel separators (ZX) will stay in the machine until sufficient operational experience has been gained with Bunch Trains to make a definitive choice of scheme for LEP2. During this evaluation period a return to Pretzel operation would therefore be possible with around three weeks of machine intervention. On removal from LEP, the ZX units will be transformed into vertical separators (ZY), and redeployed near the existing separators ZL at QS2 in the even IPs to increase the separation available for LEP2. 


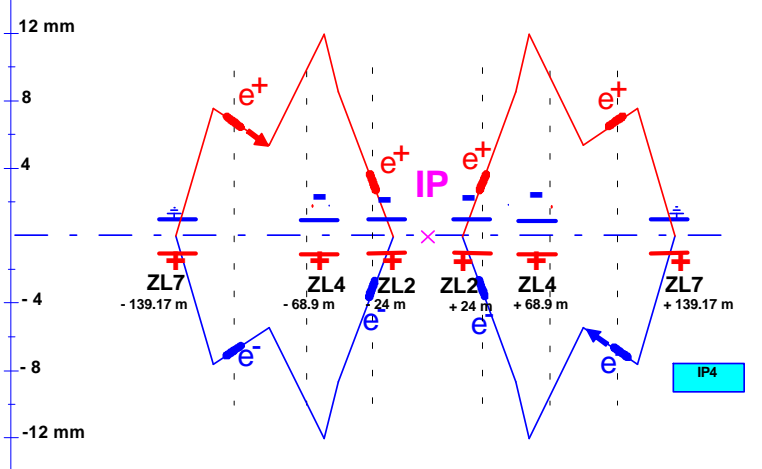

Figure 1: 1995 Bunch Train separator bump in IP4, for colliding beams.

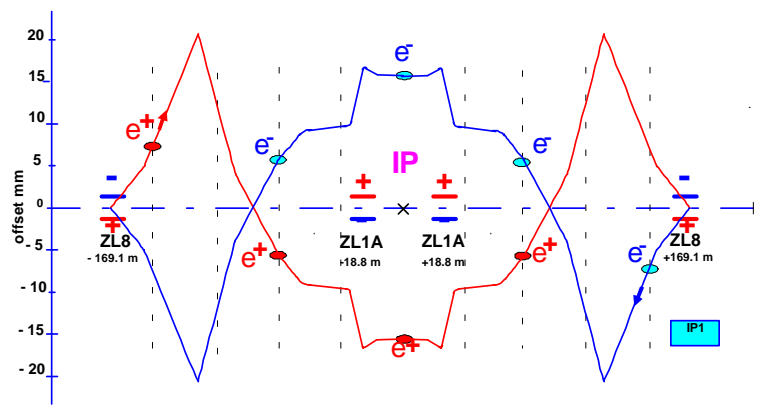

Figure 2: 1995 Bunch Train separator bump in IP1.

\section{HARDWARE CONSTRAINTS}

\section{A. Separator voltage changes}

In order to allow the bunches to collide head-on at the experimental IP, whilst maintaining the separation at the nearby parasitic encounters, all of the ZL separators in a given IP will remain powered throughout the physics datataking. This is in sharp contrast to previous years, where the beams were brought into collision by means of a fast discharge switch, which rapidly brought all the separator voltages to zero in a synchronous manner. For Bunch Train operation, the changes of separator voltage are required to be synchronous in order to maintain a properly compensated bump. This can now only be accomplished slowly (in around five minutes), by means of tables of values, since the timeconstants of the HV circuits effectively limit the rate at which the voltage can be reduced.

\section{B. Separator polarity considerations}

The polarity of the new ZL separators at QS7 in the even IP has to be positive. This is because the spark rate of both horizontal ZX and vertical ZL separators operated with negative polarity is known to be several orders of magnitude higher than for positive polarity when these separators are exposed to high synchrotron radiation fluxes $[5,6]$. In a $\mathrm{ZL}$ unit located near to the LEP arcs, the spark rate with negative polarity at a field of $30 \mathrm{kVcm}^{-1}$ was measured at $4.0 \pm 2.8 \mathrm{~h}^{-1}$, whereas for the units near to the IP operated with both negative and positive polarity the spark rate at a similar field is of the order of $4 \times 10^{-4} \mathrm{~h}^{-1}$. This effect is believed to result from the charging of insulators under the bombardment of photo-electrons, which results in a space-charge accumulation and leads to surface flashover [7].

The ZL separators are equipped with feedthroughs for both electrodes, so that the field direction can be reversed by simply connecting the HV to the opposite feedthrough. Although such a manipulation invariably requires the separator to be re-conditioned over a period of around one week, this type of change is usually made during the long annual shutdown, and can be planned accordingly, so that flexibility is retained for the choice of field direction and hence the optics.

In contrast, the vertical ZY separators to be installed at QS2 in the 1995/96 shutdown are monopolar, i.e. equipped with only one high voltage electrode, with the other electrode being connected to ground. Thus, once the ZY unit is installed, the only method of reversing the separator field direction will be to power the unit with negative high voltage. A project is now under way to develop prototype insulators which use advanced surface engineering techniques to significantly improve the $\mathrm{HV}$ performance in the presence of photon and electron fluxes [8].

\section{Vertical adjustment of collisions}

Previously, the fine 'vernier' adjustment of the vertical beam offsets to ensure head-on collisions was made using an auxiliary $\mathrm{HV}$ generator, to give a minimum step-size of better than $0.05 \mu \mathrm{m}$. For Bunch Trains this is no longer possible, as all the main generators must remain powered in order to keep the beams in collision. The average vertical offset of the train can be trimmed by adjustment of the separator voltages; the finest increment possible of around $0.4 \mu \mathrm{m}$ is imposed by the voltage resolution of the generator [9]. This is deemed adequate when compared with the expected vertical beam size of the order of $10 \mu \mathrm{m}$ and the inter-bunch spread in the vertical position of up to $7 \mu \mathrm{m}[4]$.

\section{CONTROLS}

\section{A. Initial tests in 1994}

For the initial tests in 1994, the existing hardware of the Pretzel separators was used to power the newly-installed ZL separators. Shortly before the start of the extended Bunch Train test period, the high voltage cables from the Pretzel tanks were swapped to the new ZL tanks, together with the required control cables. 
The setting of the voltage levels for the existing ZL separators was using the present Control Room (PCR) level software ('SloppySoft'). New procedures for the ramp, squeeze, collide and vernier adjustment were defined and incorporated into the control sequencer ('LEPexec'). For the new ZL separators the existing Pretzel separator 'expert software' was used, which has proved adequate since the voltage on these separators remained virtually constant. Prior to the tests proper, the initial modifications to the controls systems were commissioned and the new procedure for vernier adjustment was sucessfully tried [10].

\section{B. Operation in 1995 and beyond}

For operation in 1995 and beyond, the two new ZL separator tanks in each IP are powered by the HV supply previously used in Pretzel operation. To render the overall control system more homogeneous, the old SPS type electronics used to control this power supply has been exchanged for a controller of the type used for the vertical separators. This allows the use of the same routines from the PCR to load the tables for ramp, squeeze and collide without writing further software, although it has necessitated slight modifications in the software running in two of the equipment controllers. All other SPS type electronics previously used for the Pretzel separators has been retained.

Because of the many modifications implied by the introduction of the bunch train scheme on all levels of the controls software the opportunity was taken to migrate the process control. This was on PCs running XENIX (used previously for the vertical separators) and VME systems running under OS-9 (used for the Pretzel separators). The new location is a single PC system per IP running LynxOS. With this operation, all systems for which the hardware group is responsible now use this type of controller.

This changeover had several subsequent implications: New MIL-1553 bus controllers were used for the first time [11] to enable the desired block transfer length on LynxOS PCs. New functions to deal with the MIL-1553 message broadcast mechanism used for the vertical separators had to be added in the LynxOS MIL-1553 driver [11]. The reception and redistribution of the timing signals has been moved away from the present G-64 based hardware to a controller card in the LynxOS PCs which offers more direct access and improved monitoring facilities [11]. The 'expert software' had to be modified to deal with the PCs and to account for all modifications in the electronics (new nomenclature, addition of new equipment, removal of obsolete items). This software is used extensively for diagnostic purposes by the separator specialists and also in the PCR for non-standard controls, e.g. during special Machine Development or for commissioning of new operational procedures. It needs to be revised completely before the XENIX PCs can be physically removed since it makes heavy use of the graphics package CGI which is not available under LynxOS.

The alarm and diagnostic software has also been upgraded to take account of the changes, including the addition of new equipment and the removal of redundant items.

The high-level control of the new Bunch Train separators has been included into the 'SloppySoft' environment to properly incorporate the use of tables for bringing the beams into collision, switching between LEP hyper-runs, ensuring updated settings for vernier adjustment, allowing synchronous variation of bump amplitudes, etc. As for other software, the equipment access has been adapted to account for new controllers and redundant equipment.

\section{CONCLUSION}

The changes to the LEP electrostatic separator systems following the successful tests in 1994 [3] have been accomplished ready for the machine startup in 1995. A total of eight new separators have been installed, eight other separators have been displaced, and application software at all levels has been updated accordingly.

\section{REFERENCES}

[1] C. Bovet et al; "Final report of the 1994 Bunch Train study group", CERN SL 94-95 (AP) (1994).

[2] B. Goddard et al; "Modified LEP separator configurations for operation with Bunch Trains", CERN SL/Note 94-96 (BT) (1994).

[3] O. Brunner et al; "Experiments with Bunch Trains in LEP", this conference.

[4] E. Keil, "Offsets and crossing angles for trains of two, three and four bunches", CERN SL/Note 95-40 (AP) (1995).

[5] N. Garrel et al; "Performance Limitations in High Voltage Devices in the LEP Electron Positron Collider and its SPS Injector", Le Vide: Science, technique et applications, Suppl. to vol. 275, 386-397 (1995).

[6] W. Kalbreier and B. Goddard; "Radiation-triggered breakdown phenomena in high energy $\mathrm{e}^{+} \mathrm{e}^{-}$colliders", IEEE Trans. Elec. Insul., 28 (4), 444-453 (1993).

[7] G. Blaise and C. Le Gressus; "Charging and flashover induced by surface polarization relaxation processes", J. Appl. Phys., 69 (9) 6334-6339 (1991).

[8] B. Goddard; "Research and development to reduce beaminduced separator sparking for LEP2", CERN SL/Note 94-15 (BT) (1994).

[9] B. Balhan et al; "Separator systems for the LEP Bunch Train project", CERN SL/Note 94-64 (BT) (1994).

[10] B. Goddard et al; "Commissioning of LEP operation with Bunch Train bumps", CERN SL MD Note 160 (1994).

[11] CERN SL-CO group, various private communications. 